\title{
Differences between digit naming and number word reading in a flanker task
}

\author{
ANJA ISCHEBECK \\ University of Nijmegen, Nijmegen, The Netherlands
}

\begin{abstract}
Numbers can be represented as number words or as digits, but are the two notations processed differently? Two experiments in which a flanker paradigm with a naming task was used were conducted, with digits and number words as targets and flankers. Reaction times were shortest when the flanker denoted the same numerical value as the target. The numerical distance between the target and a numerically different flanker modulated reaction times in all conditions, except for number word targets with digit flankers. The direction of this effect- targets were named faster when the flanker was numerically close than when it was far-indicates that the numerical magnitude representations of numbers are associatively connected. When the target and the flanker were presented in the same format, no difference was observed in the distance effects for the two formats. This indicates that number words activate the abstract representation of their numerical value in a way that is very similar to that for digits.
\end{abstract}

Numbers are typically written in two distinct formats: either as Arabic digits or in the form of number words. Although these representations denote the same numeric value, they differ with respect to the manner in which they represent the pronunciation of a number. Number words consist of individual letters that represent the phonological elements of the word's pronunciation. The pronunciation of Arabic digits, on the other hand, is less determined by elements of their visual appearance. This makes it possible to represent phonologically different but semantically related numerals with the same symbol-for example, cardinal (e.g., 1 or one) or ordinal (e.g., 1st or first) numbers. The same digits are, therefore, used across many different languages.

It is an intriguing question whether these two representations are processed differently by the reader, so notationspecific effects have repeatedly been scrutinized in numerical cognition research. Digits are responded to faster than words in many numerical tasks, such as, for example, numerical comparison (Koechlin, Naccache, Block, \& Dehaene, 1999), parity judgment (Dehaene, Bossini, \& Giraux, 1993), number/nonnumber classification (Peereman $\&$ Holender, 1985), or addition and multiplication(Blankenberger \& Vorberg, 1997). However, an interaction of notation with number processing has usually not been observed (see Noël, 1991, and Holender \& Peereman, 1987, for reviews). No influence of notation has been observed, for example, on the size of the numerical distance effect

The author thanks Avishai Henik, two anonymous reviewers, Wolfgang Schwarz, and Herbert Schriefers for providing valuable comments on earlier versions of this manuscript. Correspondence concerning this article should be addressed to A. Ischebeck, Max Planck Institute for Cognitive Neuroscience, Stephanstr. 1a, 04103 Leipzig, Germany (e-mail: ischebeck@cns.mpg.de). (the time to compare two numbers decreases when their numerical distance increases; Moyer \& Landauer, 1967), the magnitude effect (number pairs are compared more quickly if they consist of small numbers; Dehaene, 1989), or the semantic congruity effect (pairs consisting of small numbers are compared more quickly if the instruction is to indicate the smaller number rather than the larger number, whereas the reverse is true for pairs consisting of large numbers; Banks, Fujii, \& Kayra-Stuart, 1976). This pattern suggests that differences between digits and number words are limited to the encoding stage, whereas all subsequent processing steps are based on a common abstract representation of the numerical value of the number.

It is often assumed that this abstract representation of the numerical value of a number is an analogue magnitude code and is stored in an orderly fashion in long-term memory like a number line (Dehaene, 1992; Dehaene, Dupoux, $\&$ Mehler, 1990; McCloskey, 1992). This assumption is based on the observation of a graded priming effect: Reaction times (RTs) to digit and number word targets decrease with their numerical distance from the prime. This has been observed across different tasks, such as numerical comparison (Dehaene, 1996; Koechlin et al., 1999; Schwarz \& Ischebeck, 2000), parity judgment (Reynvoet \& Brysbaert, 1999), and naming (Marcel \& Forrin, 1974; Reynvoet \& Brysbaert, 1999). The analogue magnitude code is assumed to be automatically activated whenever a number is encountered, because priming effects have also been observed in tasks that do not explicitly require the processing of the numerical value of a number, such as naming (Marcel \& Forrin, 1974; Reynvoet \& Brysbaert, 1999) and letter/digit classification or lexical decision (den Heyer \& Briand, 1986).

However, a more complicated pattern of results obtains when conflicting information is presented. For example, when two numbers that have to be numerically compared 
also differ in physical size of presentation on the monitor, RTs are found to increase if a comparison on the basis of physical size would lead to a different response. To illustrate, it takes longer to give a correct answer to the pair 3-5 than to the pair 3-5. This is known as the number-size congruity effect (e.g., Duncan \& McFarland, 1980; Henik \& Tzelgov, 1982). This interference effect has been reported by some investigators to be smaller or even absent for number words (Besner \& Coltheart, 1979). Another task for which a notational difference has been reported is the flanker task (Eriksen \& Eriksen, 1974). Whereas number word flankers were found to significantly influence RTs to digit targets, digit flankers had nearly no influence on number word targets (Fias, Reynvoet, \& Brysbaert, 2001). These results suggest that number words are indeed processed differently from digits in a naming task.

It is possible that some of these findings can be explained in another way. It has been observed that the amount of interference obtained depends on the processing characteristics of the conflicting information, as in the Stroop task (for a review, see MacLeod, 1991). In the case of the number-size congruity effect, the amount of interference in a numerical comparison task was found to increase with the discriminability of the physical size difference (Algom, Dekel, \& Pansky; 1996; Schwarz \& Ischebeck, in press). Because the discriminability of a fixed physical font size difference might be greater for digits than for number words consisting of several letters, interference effects might turn out to be smaller in the case of words (Foltz, Poltrock, \& Potts, 1984). Similarly, the difference observed in the number-word-digit flanker task might be due to a higher encoding speed for number words. It has also been observed that the amount of interference increases to the extent that irrelevant information is processed faster (Palef \& Olson, 1975).

Theoretical accounts about the processing architecture for numbers differ with regard to their assumptions about possible processing differences between digits and number words. Notational differences could be limited to the encoding stage or could extend to notation-specific numberprocessing routines. According to the single-format hypothesis, number words and digits are processed alike and independent of the task, once they have been translated into a common abstract representation of the numerical value (McCloskey, 1992). In this model, notational differences are assumed to be limited to a task-independent encoding stage. Notational differences might, therefore, be observed, but they should not depend on the task.

Other models (e.g., the triple-code model by Dehaene, 1992) assume task-specific processing routines that work best on a specific number format. For example, using addition and multiplication tables in long-term memory is assumed to work best with a verbal representation of numbers, whereas digits are assumed to be the preferred format of, for example, a parity judgment task. If a number is presented in a format inappropriate for the required task, it has to be translated. Notational differences are limited to a task-dependent encoding stage. This class of models, referred to here as the preferredformat hypothesis, can explain differences in RTs for the two formats in different tasks: Whereas in most numerical tasks, digits are responded to faster than words, no RT difference between the two formats has been observed in a naming task (Campbell, 1994; Ferrand, 1999).

A third class of models assumes that notational differences extend to the processing components themselves. For example, in the encoding complex model proposed by Campbell and Clark (1992), an integrated network of formand modality-specific number skills is assumed. Similarly, Koechlin et al. (1999) found a notational difference with regard to priming effects in a numerical comparison task and concluded that the representation of numerical magnitude at a very early stage might be notation specific. I will refer to this class of models as the format-specific processing hypothesis.

In the following, these three models will be contrasted. The triple-code model by Dehaene (1992) is an example of the preferred format hypothesis. Number words are assumed to be the preferred format for a naming task, and digits have to be translated. It is also assumed that a number activates its analogue magnitude code automatically in a naming task, to accommodate the observed influence of numerical distance in priming experiments with a naming task (Marcel \& Forrin, 1974).

As an example of the format-specific processing hypothesis, a modular two-pathway model based on the dual route model of reading (for a review, see Coltheart, Curtis, Atkins, \& Haller, 1993), as shown in Figure 1, is proposed. After an early visual-processing stage, the digit or the number word can be accessed in the visual mental lexicon. A naming task requires access to the phonological representation of the digit or the number word. This information can be retrieved after recognition in the lexicon, or, additionally in the case of number words, by assembling the pronunciation of the word from its constituent letters through a set of letter-to-sound conversion rules (dotted arrow). It is also assumed that a number automatically activates its analogue magnitude code after it has been recognized (double-lined arrow). This activation can interact with activation generated by a simultaneously encountered number and can influence the naming response (double-lined arrow pointing to the right).

Notational-processing differences arise in this model from the additional route to pronunciation for number words. First, it is possible that the privileged access to phonological information via the word's graphemes makes the word less susceptible to interference from a flanker digit than vice versa in a naming task. It has been assumed that number words make phonological information available earlier, as compared with other stimuli, especially in a naming task (e.g., Smith \& Magee, 1980). Second, it is also possible that the analogue magnitude code will be activated less strongly in the case of a number word, because the phonological route bypasses the lexicon and the analogue magnitude code representation. As targets, number words will be less influenced by the 


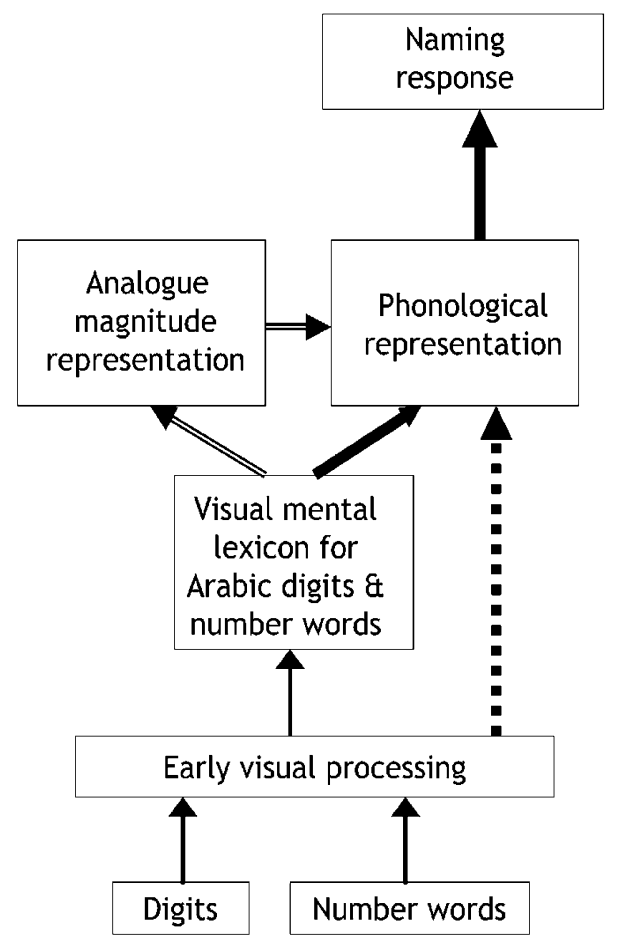

Figure 1. The two-pathway model of number naming as an example of the format-specific hypothesis. The arrow from the mental lexicon to the phonological representation indicates the direct pathway, possible for both number words and digits. The additional route, which bypasses the lexicon and is possible only for number words, is indicated by the dashed arrow. It is assumed in the model that the analogue magnitude representation of the number is automatically activated once a digit or a number word has been accessed in the mental lexicon and that this activation influences the phonological representation of the target (doublelined arrows).

numerical identity of the flanker than are digits; as flankers, their numerical identity will influence the processing of a target less strongly than will digits.

In the present study, a flanker task was conducted using numbers presented as digits or as number words. A target number was presented randomly above or below a flanker number. The participant was instructed to name the target and to ignore the flanker, which denoted the same number as or a different number from the target. The numerical distance between the flanker and the target was varied: 0 (identical), 1 (close), and 5 (far). In Experiment 1, a digit and a number word were shown, and the format of the target was fixed for the participants, who were randomly divided into two groups: Either the digit or the number word had to be named. The goal of this experiment was to investigate whether the two different formats are processed differently in a naming task.

The following predictions can be derived. According to the single-format hypothesis, both formats should influence each other similarly in a flanker task. According to the triple-code model, a larger interference effect would be expected for the more slowly encoded digit targets. The two-pathway model also predicts a larger interference ef- fect for digit targets, because the phonological information is made available more quickly by the number word flanker.

With regard to the influence of numerical distance, the triple-code model assumes a stronger effect of the flanker in the case of digit targets: The analogue magnitude code is activated earlier by the more quickly encoded number word and has more time to influence the naming of the digit than vice versa. The two-pathway model, however, does not necessarily predict a difference with regard to the effect of numerical distance, if the processing speed is similar for both formats. The numerical distance effect might be small for word targets, because they are less susceptible than digits to any influence of the numerical identity of the flanker. It might also be small for digit targets, because the analogue magnitude code is assumed to be activated less strongly by number word flankers than by digit flankers.

\section{EXPERIMENT 1 Fixed Target and Flanker Format}

\section{Method}

Participants. Twenty students at the University of Nijmegen, 16 females and 4 males, between 17 and 27 years of age, participated in the experiment. They were divided randomly into two groups. They were paid for their cooperation or received a course credit. All were native speakers of Dutch.

Materials. Each trial consisted of the simultaneous presentation of a digit and a number word, which was written in Dutch. A completely crossed design of all nine single numerals as targets and flankers was not used because numerical target-flanker distances would then be very unequally distributed and confounded with target. For example, distance 8 will be realized only with the targetflanker pairs $9-1$ and $1-9$, whereas there would be 16 target-flanker pairs for distance 1 . This is undesirable in a naming task, where a strong main effect of target, due to differences in the articulatory response, can be expected. Thus, a more restricted design was chosen: All the numerical distances used in the experiment $(0=$ identical, $1=$ close, and $5=$ far) were realized equally often and with all the targets. Care was taken that the target and the flanker provided as little information as possible about each other and their numeric distance: The 24 target-flanker pairs that met these conditions best were: $1-1,1-2,1-6,2-2,2-3,2-7,3-3,3-4,3-8,4-4,4-5,4-9$, $6-6,6-5,6-1,7-7,7-6,7-2,8-8,8-7,8-3,9-9,9-8$, and 9-4. The target digit 8 , for example, appeared with flanker number words ACHT (= eight, distance 0$)$, ZEVEN (= seven, distance 1$)$, and DRIE (= three, distance 5).

To ensure that both stimuli were read equally well and were equally likely to be read by the participant, the target was spatially separated from the flanker and appeared randomly either above or below the fixation cross (cf. Paquet \& Lortie, 1990). A single replication of the experiment consisted of 48 trials: Each target-flanker pair was presented twice, once with the target displayed above the fixation point and once below. In total, 12 replications were presented to the participants during one experimental session. The order of trials was randomized separately for each replication and each participant. For the first group of participants, the format of the targetflanker pairs was digit (target)-word (flanker); it was number word (target)-digit (flanker) for the second group of participants.

Apparatus. The stimuli were presented on a PC monitor with an $85-\mathrm{Hz}$ vertical refresh rate. Stimulus presentation was synchronized with the vertical refresh video signal. The onset of the naming response was detected by a voice key, which stopped reaction time measurement $( \pm 1 \mathrm{msec})$. Target-flanker pairs were presented in black 
on a light gray background. They were displayed in Arial font in the center of the screen, with a height of $12 \mathrm{~mm}$ for both digits and number words and with a width of $10 \mathrm{~mm}$ in the case of digits and $60 \mathrm{~mm}$ (maximum width) in the case of number words. This corresponds to a vertical viewing angle of $1^{\circ} 9^{\prime}$ and to maximum horizontal viewing angles of $57^{\prime}$ and $5^{\circ} 45^{\prime}$ for digits and number words, respectively. The target and the flanker were vertically spaced $20 \mathrm{~mm}$ (center to center) apart from each other. The participants were seated in a dimly lit room, approximately $60 \mathrm{~cm}$ from the computer screen.

Procedure. The first group of participants was instructed to name the digit and to ignore the number word; the second group of participants was instructed to name the number word and to ignore the digit. Each trial started with a small fixation cross in the middle of the screen. After a variable foreperiod with a mean of $400 \mathrm{msec}$ and a range of 300-500 msec, the fixation cross disappeared, and the target-flanker pair was displayed on the screen. A variable foreperiod was chosen so that the participants could not base their responses on an estimate of the time from the warning signal to the reaction signal (cf. Luce, 1986). The stimulus pair remained visible until time measurement was stopped by the onset of the participant's vocal response. After $1,500 \mathrm{msec}$, the next trial was presented. Within this interval, the experimenter either typed in the response of the participant or entered an error code. Errors were categorized into those made by the participant, such as wrong answers or articulatory errors, and technical errors, such as premature or delayed voice key triggering. Immediate feedback was given in the case of an error, and a practice trial was inserted before the presentation of the next experimental trial. The experiment was divided into six blocks. Breaks between blocks lasted at least $20 \mathrm{sec}$. The participants received 10 practice trials at the beginning of the experiment and 5 at the start of each new block.

\section{Results}

The first block of the experiment (two replications) was considered practice and was discarded from the analysis, which left a maximum of 480 trials per participant for analysis. Errors and invalid trials due to technical reasons were also discarded. Wrong answers (e.g., naming the flanker) and articulatory errors were combined to yield the overall error rate of the participants-in this experiment, $0.89 \%$ ( 85 trials). Another 32 trials $(0.33 \%$ ) had to be discarded due to untimely voice key triggering. An additional 3 otherwise correct trials $(0.03 \%)$ were excluded because the RT was shorter than $100 \mathrm{msec}$ or exceeded 1,000 msec. Mean RTs, standard deviations of the participant means, and error rates for the three distances are given in Table 1.

The means of the RTs for each participant in both groups were entered into a repeated measures analysis of variance (ANOVA) with the within-subjectsfactor of distance (three) and the between-subjects factor of group (two). The longer reaction times observed in the group that named digits (digit naming, $464 \mathrm{msec}$; word naming, $413 \mathrm{msec}$ ) were reflected in a significant main effect for group $[F(1,18)=$ $\left.9.29, M S_{\mathrm{e}}=4,095, p<.01\right]$. RTs were shortest for distance 0 and longest for distance 5 (428, 441, and $447 \mathrm{msec}$, for the distances 0,1 , and 5 , respectively), which yielded a significant main effect of distance $[F(2,36)=61.33$, $\left.M S_{\mathrm{e}}=28, p<.001\right]$. The interaction of group and distance was significant $\left[F(2,36)=23.28, M S_{\mathrm{e}}=28, p<.001\right]$. This was due to the greater influence of the factor of distance on the group that did digit naming than on the group that did number word naming.

For the sake of greater clarity, it is helpful to distinguish the two components that constitute the overall effect of numerical distance. The RT difference between congruent trials (distance 0: target and flanker lead to the same naming response) and incongruent trials (distance 1 and distance 5: the target and the flanker lead to different naming responses) will be called the congruency effect. The RT difference between incongruent trials with a small numerical difference between the target and the flanker (distance 1) and incongruent trials with a large numerical difference between the target and the flanker (distance 5) will be called the distance effect. Note that these two components are not independent of each other: The difference between distance 0 and the mean of the distances 1 and 5 , the difference of which is defined as the distance effect, constitutes the congruency effect. See also Table 3 for the sizes of these effects in Experiments 1 and 2.

Congruency and distance effects. A significant congruency effect $\left[F(1,18)=120.97, M S_{\mathrm{e}}=32, p<.001\right]$ was observed for the group instructed to name the digit and to ignore the number word. There was a smaller significant congruency effect $\left[F(1,18)=8.04, M S_{\mathrm{e}}=32\right.$, $p<.01]$ for the group instructed to name the number word and to ignore the digit. This was tested using contrasts (contrasts: $-2,1$, and 1 for the distances 0,1 , and 5, respectively). A significant distance effect $[F(1,18)=$ 25.27, $\left.M S_{\mathrm{e}}=24, p<.001\right]$ was observed for the group naming digits, but not for the group naming words (1 msec, n.s.). Target digits were named significantly more quickly when the flanker word was numerically close to the target (distance 1) than when it was numerically far (distance 5). This was tested using contrasts (contrast: $0,-1$, and 1 for the distances 0,1 , and 5 , respec-

Table 1

Experiment 1: Mean Reaction Times (in Milliseconds, With Standard Deviations) and Error Rates (\% E) as a Function of Condition

\begin{tabular}{|c|c|c|c|c|c|c|c|c|c|}
\hline \multirow[b]{3}{*}{ Group } & \multicolumn{9}{|c|}{ Distance } \\
\hline & \multicolumn{3}{|c|}{0} & \multicolumn{3}{|c|}{1} & \multicolumn{3}{|c|}{5} \\
\hline & $M$ & $S D$ & $\overline{\% \mathrm{E}}$ & $M$ & $S D$ & $\% \mathrm{E}$ & $M$ & $S D$ & $\% \mathrm{E}$ \\
\hline Digit-word & 448 & 38.5 & 0.06 & 466 & 37.9 & 2.00 & 477 & 41.8 & 2.50 \\
\hline Word-digit & 409 & 33.4 & 0.06 & 415 & 36.1 & 0.56 & 416 & 34.9 & 0.31 \\
\hline
\end{tabular}

Note-Digit-word, digit target and word flanker; word-digit, word target and digit flanker. 
tively). The difference between the two groups was significant with regard to the size of the congruency effect $\left[F(1,18)=32.81, M S_{\mathrm{e}}=32, p<.001\right]$, as well as with regard to the size of the distance effect $[F(1,18)=10.68$, $\left.M S_{\mathrm{e}}=24, p<0.01\right]$. Number word targets were named $39 \mathrm{msec}$ more quickly than digit targets in congruent trials [distance $0 ; F(1,18)=5.70, M S_{\mathrm{e}}=1,301, p<.05$ ]. The RT differences found for the factor of distance cannot be attributed to a speed-accuracy tradeoff, because the longer RTs for the greater distances were also accompanied by higher error rates: In the digit naming group 0,31 , and 39 errors were made in total at the distances 0,1 , and 5, respectively. In the group number naming groups, the participants made 8,5 , and 1 errors, respectively.

\section{Discussion}

The presence of a congruency effect for both groups clearly shows that the numerical identity of the digit or number word flanker influenced the response of the participant. RTs were significantly faster when the target and the flanker denoted the same number and led to the same naming response (distance 0 ). The congruency effect was significantly greater for digit targets with word flankers than for word targets with digit flankers.

A significant distance effect was observed, but only in the case of digit targets, which were named more quickly in the presence of numerically close (distance 1) flankers than in the presence of numerically far (distance 5) flankers. The difference between the two groups with regard to the size of this effect was also significant. The presence of a distance effect for digit targets indicates that number word flankers activated the abstract representation of their numerical value. This activation spread to neighboring nodes, including the target, which facilitated the digit naming response. The direction of the distance effect (shorter RTs for numerically close flankers) is the same as that observed in priming experiments (Marcel \& Forrin, 1974).

The greater congruency and distance effects for digit targets are compatible with the preferred format hypothesis, as long as they can be attributed to a difference between the encoding speeds of the two formats. Such a difference is indicated by the significant difference between the RTs for the two target types in congruent trials (distance 0; e.g., TwO-2 vs. 2-TWO): Number words were named more quickly than digit targets. The more quickly encoded number word flankers have a greater opportunity to influence the digit naming response than vice versa.

The greater congruency effect for digit targets is compatible with the two-pathway model, which assumes that the additional route makes number words less susceptible to interference. However, the model did not predict a greater distance effect for digit targets with word flankers, unless a difference in processing speed between the two formats is also assumed. Because number words are assumed to activate the magnitude code to a lesser degree than do digits, a word flanker will influence a digit target less strongly than will a digit flanker. Similarly, a word target will be less vulnerable than a digit target to the numerical identity of a digit flanker. For similar encoding speeds, similar and small distance effects for both mixed-format conditions can be predicted. If encoding speeds differ, as indicated by the difference between digits and number words in congruent trials, the predictions of the two-pathway model will be similar to those derived from the preferred format hypothesis. It is, therefore, difficult to decide between these two models on the basis of this experiment alone.

It is possible, however, that the difference between congruent trials in the two format conditions is due to strategic effects, given that format was presented blocked. In the case of number words, the participants might have made use of the knowledge that the target was always a word and, for example, used exclusively the assembly route. Target format was also varied between participants, so the observed shorter reaction times to word targets might have been due to interindividual differences.

It was therefore advisable to test the predictions outlined earlier in a second experiment in which format was varied randomly from trial to trial for each participant. In Experiment 2, the target and the flanker were distinguished by color and were presented in all four format combinations: digit-word, word-digit, word-word, and digit-digit (with labels in the target-flanker order). Predictions were tested that were similar to those in Experiment 1. First, the result pattern should not depend on format if both formats are encoded and processed similarly. If both formats are processed similarly but encoded with different speeds, as assumed by the preferred format hypothesis, the following result pattern should obtain: Distance and congruency effects should be minimal for number word targets and digit flankers, they should be similar for pure format trials (word-word and digit-digit), and they should be maximal for digit targets and number word flankers.

The two-pathway model, if the assumption of similar encoding speeds were dropped, would also predict an asymmetric pattern of results for the mixed-format conditions. In addition, a specific prediction of the two-pathway model with regard to the pure-format conditions can be derived: If number words are read partly by using the additional route that bypasses the lexicon, number words should activate the representation of their numerical value less strongly than do digits. The distance effect, which is related solely to the interaction of the activation at this level between the target and the flanker, should therefore be smaller for word-word than for digit-digit trials.

\section{EXPERIMENT 2 Variable Target and Flanker Format}

\section{Method}

Participants. Twenty students at the University of Nijmegen, 5 males and 15 females, between 19 and 28 years of age, participated in the experiment. They were paid for their cooperation or received course credit. All were native speakers of Dutch. No participant had taken part in Experiment 1.

Materials. The materials and design in Experiment 2 were identical to those in Experiment 1, with the following exceptions: The notation in which the target and the flanker appeared on the screen 
now varied randomly between trials. Color was used as a cue, with the target presented in black and the flanker in red. Given that all four possible format combinations were used in this experiment, one full replication of the experiment now consisted of 192 trials (24 targetflanker pairs, two positions, and four format combinations).

Apparatus and Procedure. The procedure and the apparatus were identical to those in Experiment 1, except with regard to the presentation of the stimuli and the instructions. Two measures were taken to magnify possible interference effects: (1) The targets were printed in black, the flankers in red, and (2) spatial uncertainty was increased by adding a random horizontal shift (maximum $\pm 5 \mathrm{~mm}$ on the screen) to the vertical spatial uncertainty. The participants were instructed to name the number printed in black and to ignore the red flanker. Four replications were obtained from each participant during one session. The experiment was divided into eight blocks and took approximately $45 \mathrm{~min}$.

\section{Results}

The first two blocks (one replication) of the experiment were considered practice and were discarded from the analysis. This yielded a maximum of 576 experimental trials per participant for data analysis. The participants made 67 errors $(0.58 \%)$ in total; 26 trials $(0.23 \%)$ had to be discarded due to technical problems. Again, RTs shorter than $100 \mathrm{msec}$ and longer than $1,000 \mathrm{msec}$ in otherwise correct trials were discarded, which resulted in the omission of seven additional data points $(0.06 \%)$. Mean RTs, standard deviations of the participant means, and error rates for the three distances are given in Table 2.

The means of the RTs for each participant in every experimental condition were entered into a repeated measures ANOVA with the three within-subjects factors of target format (two), flanker format (two), and distance (three). The comparatively longer reaction times for digit targets than for word targets (483 and $468 \mathrm{msec}$ ), as well as for word flankers, as compared with digit flankers (486 and $465 \mathrm{msec}$ ), are reflected in the significant main effects of the factors of target format $\left[F(1,19)=48.70, M S_{\mathrm{e}}=270\right.$, $p<.001]$ and flanker format $\left[F(1,19)=311.50, M S_{\mathrm{e}}=\right.$ $88, p<.001]$. RTs to congruent trials (distance 0 ) were again shortest $(459 \mathrm{msec})$, followed by the mean for distance $1(477 \mathrm{msec})$, and they were longest for distance 5 (490 msec), which led to a significant main effect for the factor of distance $\left[F(2,38)=141.74, M S_{\mathrm{e}}=138, p<\right.$ $.001]$. The observed significant interaction between the factors of target format and flanker format $[F(1,19)=$ $\left.7.00, M S_{\mathrm{e}}=131, p<.05\right]$ indicates that their effects were not additive: A digit flanker slowed the reaction time to a digit target more than that to a number word target. Both factors were found to interact with the factor of distance separately $\left[F(2,38)=8.89, M S_{\mathrm{e}}=81, p<.001 ; F(2,38)=\right.$ $25.53, M S_{\mathrm{e}}=73, p<.001$ ], as well as jointly [triple interaction: $\left.F(2,38)=9.96, M S_{\mathrm{e}}=62, p<.001\right]$. This was due to the differences between congruency and distance effects for the four format combinations (see Table 3 for the respective sizes and significance levels of these effects).

Distance and congruency effects. Only a small but significant congruency effect was observed when the target was a number word and the flanker was a digit $[F(1,19)=$ $\left.12.21, M S_{\mathrm{e}}=90, p<.01\right]$. Significant congruency effects were observed for the three other target-flanker format combinations-namely, digit-digit $\left[F(1,19)=70.75, M S_{\mathrm{e}}=\right.$ $121, p<.001]$, digit-word $\left[F(1,19)=91.89, M S_{\mathrm{e}}=136\right.$, $p<.001]$, and word-word, $\left[F(1,19)=125.48, M S_{\mathrm{e}}=119\right.$, $p<.001]$. This was tested using contrasts. Significant distance effects were found for the same three format combinations [digit-digit, $F(1,19)=38.61, M S_{\mathrm{e}}=50, p<.001$; digit-word, $F(1,19)=68.96, M S_{\mathrm{e}}=65, p<.001$; wordword, $\left.F(1,19)=29.98, M S_{\mathrm{e}}=62, p<.001\right]$. No distance effect (n.s., $p>.23$ ) was observed for number word targets with digit flankers. Similar to the results in Experiment 1 , RTs to digit targets flanked by number words in congruent trials (distance 0 ) were significantly longer than those in the other three format combinations $(p<$ .001 , tested using Scheffé-tests), which did not differ from each other. The RT differences found for the factor of distance were accompanied by higher error rates and can, therefore, not be attributed to a speed-accuracy tradeoff: The participants made 5, 22, and 36 errors in total, at the distances 0,1 , and 5, respectively (digit-digit, 2, 2, and 11 ; digit-word, 2, 10, and 8; word-digit, 1, 4, and 2; wordword, 0,6 , and 15).

\section{Discussion}

With regard to the mixed-format conditions (worddigit and digit-word), the results of Experiment 2 replicated the results of Experiment 1. For number word targets and digit flankers, only a small congruency effect and no distance effect obtained, whereas significant congruency and distance effects were observed for digit targets and

Table 2

Experiment 2: Mean Reaction Times (in Milliseconds, With Standard Deviations) and Error Rates (\% E) as a Function of Condition

\begin{tabular}{|c|c|c|c|c|c|c|c|c|c|}
\hline \multirow[b]{3}{*}{ Flanker } & \multicolumn{9}{|c|}{ Distance } \\
\hline & \multicolumn{3}{|c|}{0} & \multicolumn{3}{|c|}{1} & \multicolumn{3}{|c|}{5} \\
\hline & $M$ & $S D$ & $\% \mathrm{E}$ & $M$ & $S D$ & $\% \mathrm{E}$ & $M$ & $S D$ & $\% \mathrm{E}$ \\
\hline \multicolumn{10}{|c|}{ Target: Digit } \\
\hline Digit & 453 & 33.8 & 0.31 & 472 & 34.7 & 0.21 & 485 & 36.5 & 1.15 \\
\hline Word & 475 & 40.6 & 0.42 & 495 & 38.1 & 1.04 & 516 & 36.3 & 1.04 \\
\hline \multicolumn{10}{|c|}{ Target: Word } \\
\hline Digit & 453 & 40.9 & 0.21 & 461 & 40.1 & 0.52 & 464 & 38.8 & 0.42 \\
\hline Word & 454 & 37.7 & 0.00 & 481 & 37.1 & 0.63 & 495 & 39.8 & 1.77 \\
\hline
\end{tabular}


Table 3

Experiments 1 and 2: Size (in Milliseconds) and Significance Level of the Congruency and Distance Effects

\begin{tabular}{ccc}
\hline Format & Congruency Effect & Distance Effect \\
\hline & Experiment 1 & \\
Digit-word & $24^{* *}$ & $11^{* *}$ \\
Word-digit & $6^{* * *}$ & $1 * * *$ \\
& Experiment 2 & \\
Digit-digit & $25^{* *}$ & $14^{* *}$ \\
Digit-word & $31^{* *}$ & $21^{* *}$ \\
Word-digit & $9 *$ & $3^{* * *}$ \\
Word-word & $33^{* *}$ & $14^{* *}$ \\
\hline
\end{tabular}

Note-The level of significance was calculated using contrasts. Congruency effect, difference between the mean of the reaction times for distances 5 and 1 and distance 0 ; distance effect, difference between the reaction times for distance 5 and distance 1; format, format of target and flanker given in the target-flanker order. $* p$ values are Bonferronicorrected for 12 comparisons. $* p<.05$. **p $<.01$. ***p $<.001$.

number word flankers. RTs were significantly longer in the congruent condition (distance 0 ) for digit targets with number word flankers than in the three other format combinations. In the pure-format conditions (word-word and digit-digit), significant congruency and distance effects were observed for word targets, as well as for digit targets. There was no difference with regard to the size of the distance effect for word-word and digit-digit trials. A distance effect was observed in three out of four format combinations, and it had the same direction as that in Experiment 1: RTs were faster when the flanker was numerically close to the target.

The similarly sized distance effects in the pure-format conditions do not support the two-pathway model. If number words are read over the additional route, they should not activate the numerical magnitude code as strongly as do digits, because the lexicon is bypassed. This should then lead to a smaller distance effect in the wordword condition than in the digit-digit condition.

Given that the two formats are encoded at different speeds, the observed results for the four format combinations are compatible with the predictions derived from the preferred format hypothesis. In the case of the more quickly processed number word target, a digit flanker has little opportunity to influence the naming response, which explains the very small congruency and absent distance effects observed for the word-digit format combination in Experiments 1 and 2. When the target and the flanker do not differ with regard to processing speed, as in the pureformat conditions, equally large congruency and distance effects can be expected and have been observed. In the case of a digit target and a number word flanker, the largest congruency and distance effects are expected and observed, because the more quickly processed flanker has more time to interfere with the more slowly processed target.

It should be noted, however, that only the format combination of a digit target with a number word flanker provides evidence for a difference in encoding speeds. No difference, however, was observed between digit-digit and word-word trials. Together with the earlier finding of similar naming times for digits and number words when presented alone (Campbell, 1994; Ferrand, 1999), this observation makes it difficult to uphold the assumption of similar encoding speeds for the two formats. It is possible that digit targets might take longer to name only in the presence of number words.

\section{GENERAL DISCUSSION}

The main goal of the present study was to investigate possible processing differences between number words and digits in a naming task. According to the single-format hypothesis, which assumes that both formats are processed similarly and equally quickly, the results of both experiments should be independent of the format of target and flanker number. This was not observed. Whereas strong congruency and distance effects were found in the case of digit targets and number word flankers, there was only a small congruency effect and no distance effect for number word targets in the presence of digit flankers. In the pureformat trials (word-word and digit-digit), on the other hand, the observed congruency and distance effects were of similar size.

The format-specific processing hypothesis, as specified in the two-pathway model, also cannot account for the results. In this model, it is assumed that notational processing differences are due to format-specific processing routines for naming. The additional phonological assembly route assumed in the two-pathway model for number words bypasses the lexicon and, therefore, leads to a reduced automatic activation of the magnitude code, as compared with digits. The distance effect, which stems from the interaction on the level of the magnitude representation, is therefore expected to be larger for digit-digit trials than for word-word trials. However, equal distance effects were observed in the pure-format trials. This suggests that the two formats do not activate their numerical magnitude representations differently. This finding does not run counter to the dual-route model of reading (Coltheart et al., 1993), which assumes, for words with a high frequency of occurrence, that the direct visual route to the lexicon outperforms the phonological assembly route. Number words from one to nine have indeed a high frequency of occurrence in Dutch-between 50 and 1,000 per million, according to the Celex Dutch language database (Baayen, Piepenbrock, \& van Rijn, 1993). However, in this case, the predictions of the two-pathway model would be similar to the predictions derived from the preferred format hypothesis: The numerical identity of number words and digits is processed similarly, but number words and digits might influence each other asymmetrically, due to different encoding speeds.

Given a difference in encoding speeds, the observed pattern of results is compatible with the preferred format hypothesis. As formulated in the triple-code model, the preferred format for a naming task is verbal, and digits are translated before they are named. If number words are en- 
coded more quickly than digits, the greatest congruency and distance effects can be expected when a quickly processed number word flanker interacts with a more slowly processed digit target, whereas only small congruency and distance effects are expected for word targets with digit flankers. If the target and the flanker are processed equally quickly, as in the pure-format trials, congruency and distance effects should not differ. The results of Experiments 1 and 2 match these predictions.

However, a part of the results pattern does not fit this account: RTs in the digit-digit trials should also be longer than those in the word-word trials if words are encoded more quickly than digits, but this was not observed. Together with the observation that number words are not named more quickly than digits when presented without a flanker (Campbell, 1994; Ferrand, 1999), this is difficult to reconcile with the assumption of a faster encoding speed for number words. Longer RTs have been observed for digits only when a word flanker was present. The preferred format hypothesis, however, cannot account for the results if encoding speeds do not differ.

One possible way to save the preferred format hypothesis is to assume that the amount of interference does not depend solely on the relative difference between the encoding speeds of the two formats. Although the relative speed of processing hypothesis has the advantage of being very straightforward and accounting for numerous results of interference experiments (Dyer, 1973), there is also ample evidence that this account alone is not sufficient. For example, when word reading was slowed considerably by presenting the words upside down and backward, incongruent words were still observed to interfere with color naming (Dunbar \& MacLeod, 1984). Alternative accounts include the assumption of a greater degree of automaticity (MacLeod \& Dunbar, 1988) or response specificity (Smith \& Magee, 1980) in the case of number words, as compared with other stimuli in a naming task. This advantage for words makes them less vulnerable to any information provided by a simultaneously presented digit, whereas the naming of digits might be greatly influenced by number words.

An additional interesting finding of the present study was the observed facilitating influence of close numerical distances between a target and a flanker, as compared with flankers numerically far from the target. The observed direction of the distance effect corresponds very well to the results of priming experiments with digits (Marcel \& Forrin, 1974) and digits and number words (Reynvoet \& Brysbaert, 1999). However, other studies have suggested that RTs to a number target could have been expected to be longer in the presence of numerically close flankers, as compared with numerically far flankers.

For example, a different direction is suggested by the numeric distance effect, which has also been attributed to the activation of the analogue magnitude code: In a comparison task, RTs are usually observed to be longer for close numerical distances (Moyer \& Landauer, 1967). This effect has also been reported for same-different judgments of numbers, which do not require an explicitsmaller- larger judgment (Dehaene \& Akhavein, 1995), and for other semantic comparison tasks involving nonnumerical stimuli (Moyer \& Bayer, 1976). A facilitatory effect for numerically close numbers was again observed in a comparison task when a target number was primed by another numeral (e.g., Koechlin et al., 1999).

More difficult to reconcile with the direction found in the present study, however, are the results of other interference experiments using naming tasks, in which a target is presented together with conflicting information related to the meaning of the target. Usually, longer RTs are observed when semantically related conflicting information is presented. This is not compatible with the present results if numerical distance is apprehended as semantic dissimilarity. In a Stroop task, it takes less time to name the print color of a word unrelated to a color than of a word denoting a color different from the required response (Fox, Shor, \& Steinman, 1971; Klein, 1964). In picture-word interference experiments, longer RTs have been observed in a picture-naming task for semantically related words than for semantically unrelated words (Lupker, 1979; Rosinski, 1977). Pavese and Umiltà $(1998,1999)$ also found a small but consistent inhibitory effect for close numerical distances in a digit numerosity naming task.

However, this pattern of results is not observed with perfect consistency. For example, a study by Dallas and Merikle (1976), using written words in a flanker task, reported a semantic distance effect with a direction similar to the results of the present study. Flowers, Nelson, Carson, and Larsen (1984) reported longer RTs for digit flankers with numerical values nonadjacent to the target, as compared with flankers with adjacent numerical values. Similarly, inhibitory priming effects have been observed by MacDonald and Joordens (2000) for a subset of trials in a number word naming experiment. It is, therefore, possible that semantically related flankers give rise to inhibitory and facilitatory effects, only the net result of which is experimentally observed.

To conclude, the results of the present experiments indicate that number words and digits activate their abstract numerical magnitude representation similarly and that processing differences between digits and number words in a flanker task are limited to the encoding stage. However, these differences might include a greater automaticity or response specificity for number words in a naming task, rather than a difference in encoding speeds.

\section{REFERENCES}

Algom, D., Dekel, A. \& Pansky, A. (1996). The perception of number from the separability of the stimulus: The Stroop effect revisited. Memory \& Cognition, 24, 557-572.

Batyen, R. H., Piepenbrock, R., \& van Rijn, H. (1993). The Celex lexical database [CD-ROM]. Philadelphia: University of Pennsylvania, Linguistic Data Consortium.

Banks, W. P., FujII, M., \& KaYra-Stuart,F. (1976). Semantic congruity effects in comparative judgments of magnitudes of digits. Journal of Experimental Psychology: Human Perception \& Performance, 2, 435-447.

Besner, D., \& Coltheart, M. (1979). Ideographic and alphabetic processing in skilled reading of English. Neuropsychologia, 17, 467-472.

Blankenberger,S., \& Vorberg, D. (1997). The single-format assump- 
tion in arithmetic fact retrieval. Journal of Experimental Psychology: Learning, Memory, \& Cognition, 23, 721-738.

CAmpbell, J. I. D. (1994). Architectures for numerical cognition. Cognition, 53, 1-44

CAmpbell, J. I. D., \& Clark, J. M. (1992). Cognitive number processing: An encoding-complex perspective. In J. I. D. Campbell (Ed.), The nature and origins of mathematical skills (pp. 457-491). Amsterdam: Elsevier, North-Holland.

Coltheart, M., Curtis, B., Atkins, P., \& Haller, M. (1993). Models of reading aloud: Dual-route and parallel-distributed-processing approaches. Psychological Review, 100, 589-608.

Dallas, M., \& Merikle,P. (1976). Semantic processing of non-attended visual information. Canadian Journal of Psychology, 30, 15-21.

DehaEne, S. (1989). The psychophysics of numerical comparison: A reexamination of apparently incompatible data. Perception \& Psychophysics, 45, 557-566.

Dehaene, S. (1992). Varieties of numerical abilities. Cognition, 44, 1-42.

Dehaene, S. (1996). The organization of brain activations in number comparison: Event-related potentials and the additive factors method. Journal of Cognitive Neuroscience, 8, 47-68.

Dehaene, S., \& AKhavein, R. (1995). Attention, automaticity, and levels of representation in number processing. Journal of Experimental Psychology: Learning, Memory, \& Cognition, 21, 314-326.

Dehaene, S., Bossini, S., \& Giraux, P. (1993). The mental representation of parity and number magnitude. Journal of Experimental Psychology: General, 122, 371-396.

Dehaene, S., Dupoux, E., \& Mehler, J. (1990). Is numerical comparison digital? Analogueical and symbolic effects in two-digit number comparison. Journal of Experimental Psychology: Human Perception \& Performance, 16, 626-641.

den Heyer, K., \& Briand, K. (1986). Priming single digit numbers: Automatic spreading activation dissipates as a function of semantic distance. American Journal of Psychology, 99, 315-340.

Dunbar, K., \& MacLeod, C. M. (1984). A horse race of a different color: Stroop interference patterns with transformed words. Journal of Experimental Psychology: Human Perception \& Performance, 10, 622-639.

Duncan, E. M., \& McFarland, C. E., JR. (1980). Isolating the effects of symbolic distance and semantic congruity in comparative judgments: An additive-factors analysis. Memory \& Cognition, 8, 612622.

DYER, F. N. (1973). The Stroop phenomenon and its use in the study of perceptual, cognitive, and response processes. Memory \& Cognition, 1, 106-120.

ERIKSEN, B. A., \& ERIKSEN, C. W. (1974). Effects of noise letters upon the identification of a target letter in a nonsearch task. Perception \& Psychophysics, 16, 143-149.

FERRAND, L. (1999). Why naming takes longer than reading? The special case of Arabic numbers. Acta Psychologica, 100, 253-266.

Fias, W., Reynvoet, B., \& Brysbaert, M. (2001). Are Arabic numerals processed as pictures in a Stroop interference task? Psychological Research, 65, 242-249.

Flowers, J. H., Nelson, S. M., Carson, D., \& Larsen, L. (1984). Automatic and expectancy-based priming effects in a digit naming task. Journal of Experimental Psychology: Human Perception \& Performance, 10, 65-74.

Foltz, G. S., Poltrock, S. E., \& Potts, G. R. (1984). Mental comparison of size and magnitude. Journal of Experimental Psychology: Learning, Memory, \& Cognition, 10, 442-453.

Fox, L. A., Shor, R. E. \& Steinman, R. J. (1971). Semantic gradients and interference in naming color, spatial direction and numerosity. Journal of Experimental Psychology, 91, 59-65.

HeniK, A., \& Tzelgov, J. (1982). Is three greater than five: The relation between physical and semantic size in comparison tasks. Memory \& Cognition, 10, 389-395.

Holender, D., \& Peereman, R. (1987). Differential processing of phonographic and logographic single-digit numbers by the two hemi- spheres. In G. Deloche \& X. Seron (Eds.), Mathematical disabilities (pp. 43-85). Hillsdale, NJ: Erlbaum.

KLEIN, G. S. (1964). Semantic power measured through the interference of words with color-naming. American Journal of Psychology, 77, 576-588.

Koechlin,E., Naccache,L., Block, E., \& Dehaene, S. (1999). Primed numbers: Exploring the modularity of numerical representations with masked and unmasked semantic priming. Journal of Experimental Psychology: Human Perception \& Performance, 25, 1882-1905.

LuCE, R. D. (1986). Reaction times: Their role in inferring elementary mental organization. New York: Oxford University Press.

LUPKER, S. J. (1979). The semantic nature of response competition in the picture-word interference task. Memory \& Cognition, 7, 485-495.

MacDonald, P. A., \& Joordens, S. (2000). Investigating a memorybased account of negative priming: Support for selection-feature mismatch. Journal of Experimental Psychology: Human Perception \& Performance, 26, 1478-1496.

MacLeod, C. M. (1991). Half a century of research on the Stroop effect: An integrative review. Psychological Bulletin, 109, 163-203.

MacLeod, C. M., \& Dunbar, K. (1988). Training and Stroop-like interference: Evidence for a continuum of automaticity. Journal of Experimental Psychology: Learning, Memory, \& Cognition, 14, 126-135.

MarCel, T., \& Forrin, B. (1974). Naming latency and the repetition of stimulus categories. Journal of Experimental Psychology, 103, 450-460.

MCCLOSKEY, M. (1992). Cognitive mechanisms in numerical processing: Evidence from acquired dyscalculia. Cognition, 44, 107-157.

Moyer, R. S., \& BAYER, R. H. (1976). Mental comparison and the symbolic distance effect. Cognitive Psychology, 8, 228-246.

Moyer, R. S., \& LANDAUER, T. K. (1967). Time required for judgments of numerical inequality. Nature, 215, 1519-1520.

NoËL, M.-P. (1991). Influence des systèmes de notation des nombres sur les méchanismes d'encodage et de traitement numériques [Notational systems for numbers: Impact on the encoding mechanisms and numerical processes]. L'Année Psychologique, 91, 581-607.

Palef, S. R. \& Olson, D. R. (1975). Spatial and verbal rivalry in a Strooplike task. Canadian Journal of Psychology, 29, 201-209.

Paquet, L., \& Lortie, C. (1990). Evidence for early selection: Precuing target location reduces interference from same-category distractors. Perception \& Psychophysics, 48, 382-388.

PAvese, A., \& UmiLtà, C. (1998). Symbolic distance between numerosity and identity modulates Stroop interference. Journal of Experimental Psychology: Human Perception \& Performance, 24, 1535-1545.

Pavese, A., \& Umiltà, C. (1999). Further evidence on the effects of symbolic distance on Stroop-like interference. Psychological Research, 62, 62-71.

Peereman, R, \& Holender, D. (1985). Visual field differences for a number-non-number classification of alphabtic and ideographic stimuli. Quarterly Journal of Experimental Psychology, 36A, 197-216.

Reynvoet, B., \& Brysbaert, M. (1999). Single-digit and two-digit Arabic numerals address the same semantic number line. Cognition, 72, 191-201.

RosinsKi, R. R. (1977). Picture-word interference is semantically based. Child Development, 48, 643-647.

Schwarz, W., \& Ischebeck, A. (2000). Sequential effects in number comparison. Journal of Experimental Psychology: Human Perception \& Performance, 26, 1606-1621.

SCHWARZ, W., \& IschebecK, A. (in press). On the relative speed account of number size interference in comparative judgments of numerals. Journal of Experimental Psychology: Human Perception \& Performance.

SMith, M. C., \& MAgEe, L. E. (1980). Tracing the time course of pictureword processing. Journal of Experimental Psychology: General, 109, 373-392.

(Manuscript received April 2, 2001; revision accepted for publication March 5, 2003.) 